\title{
Treatment of orbital vascular malformations with intralesional injection of pingyangmycin
}

\author{
Han Yue, ${ }^{1}$ Jiang Qian, ${ }^{1}$ Victor M Elner, ${ }^{2}$ Jie Guo, ${ }^{1}$ Yi-Fei Yuan, ${ }^{1}$ Rui Zhang, ${ }^{1}$ Qin Ge ${ }^{1}$
}

- Additional material is published online only. To view please visit the journal online (http://dx.doi.org/10.1136/ bjophthalmol-2012-302900).

${ }^{1}$ Department of

Ophthalmology, Eye and ENT Hospital of Fudan University, Shanghai, China

${ }^{2}$ Department of Ophthalmology and Visual Sciences and Department of Pathology, University of Michigan, Ann Arbor, Michigan, USA

\section{Correspondence to} Dr Jiang Qian, Department of Ophthalmology, Eye and ENT Hospital of Fudan University, 83 Fenyang $\mathrm{Rd}$

Shanghai 200031, China; qianjiang58@hotmail.com

Received 17 November 2012 Revised 6 March 2013 Accepted 20 March 2013 Published Online First 19 April 2013

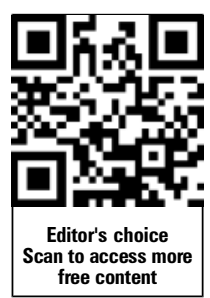

To cite: Yue H, Qian J, Elner VM, et al. $\mathrm{Br} J$ Ophthalmol 2013:97: 739-745.

\section{ABSTRACT}

Background To evaluate the safety and efficacy of intralesional pingyangmycin for treatment of orbital vascular malformations.

Methods Thirteen patients received intralesional injections of pingyangmycin at the Eye and ENT Hospital of Fudan University. CT scans of each patient were evaluated with graphic processing software to measure lesion volumes before and after treatment.

Results of five men and eight women patients averaging 36 years old, six were diagnosed with venous malformations, six with cavernous haemangiomas, and one with lymphangioma. Proptosis and eyelid swelling were the most common presenting signs. The mean preoperative lesion volume was $4.4 \pm 2.3 \mathrm{~cm}^{3}$ (range, $1.6-8.8 \mathrm{~cm}^{3}$ ), and the average postoperative volume was $1.5 \pm 1.7 \mathrm{~cm}^{3}$ (range, $0.2-6.6 \mathrm{~cm}^{3}$ ) after a single pingyangmycin injection, the dose of which averaged $4.2 \mathrm{mg}$ (range, 2-6 mg). The mean percentage of volume reduction was $70.0 \%$ (range, $24.5-88.3 \%$ ), whereas proptosis reduction averaged $3.2 \mathrm{~mm}$ (range, $0-5.5 \mathrm{~mm}$ ). The lesion volume and proptosis reductions were both statistically significant $(p<0.001$, and $p=0.001$ ). No adverse local or systemic side effects occurred in any of the patients during follow-up averaging 13.3 months. Histopathology of a cavernous haemangioma that underwent a second injection through a surgical incision, showed a mild chronic inflammatory response, increased numbers of myofibroblasts, loss of vascularity and fibrosis, all consistent with known effects of bleomycin treatment. Conclusions Intralesional injection of pingyangmycin for the treatment of orbital vascular malformations is an effective method that involves mild inflammation, fibrosis, and reduced vascularity of the malformation.

\section{INTRODUCTION}

Vascular malformations are among the most common space-occupying lesions of the orbit. They often result in proptosis, pain, swelling, motility disturbances, visual impairment and disfigurement. Orbital vascular malformations comprise a wide spectrum of abnormalities, which may exhibit various combinations of venous, arterial or lymphatic differentiation. Currently, orbital vascular abnormalities are treated in a multidisciplinary fashion, including surgical excision, selective embolisation, laser therapy, sclerotherapy, etc. ${ }^{1}$ The therapeutic choice for each patient depends on the site and size of lesions, hemodynamics, type of abnormality, and surgeon preference.

The treatment of orbital vascular malformations remains challenging because many are difficult to surgically resect due to their sheer size and their permeation of orbital structures, including extraocular muscles, motor and sensory nerves, and the optic nerve. They are particularly difficult to resect when located deep in the orbital apex where excision may be complicated by severe haemorrhage in addition to damage to the aforementioned structures. Lasers are only appropriate for superficial malformations whereas radiotherapy is a poor choice due to the relative resistance of these lesions to radiotherapy, radiotoxicity to normal structures, and cosmetic complications. Embolisation also has significant drawbacks due to potential infarction of normal structures with loss of visual function, inconsistent efficacy and shunting of flow following embolisation. $^{2}$

Because of these drawbacks, direct puncture sclerotherapy has been proposed as a minimally invasive treatment option for these cases. ${ }^{3}{ }^{4}$ Of the available sclerosing agents, pingyangmycin (bleomycin A5) has proved to be safe and effective in the treatment of vascular malformations of the head and neck. ${ }^{5-8}$ We report our experience using intralesional pingyangmycin treatment for orbital venous and lymphatic malformations, including orbital cavernous haemangiomas. ${ }^{9} 10$ To measure the efficacy in a quantitative fashion, we analysed preinjection and postinjection CT scans with graphic processing software to measure lesional volume reduction after a single injection of pingyangmycin. In one case, we also examined the histopathologic features of a cavernous haemangioma following injection with the drug.

\section{METHODS}

\section{Patient and diagnosis}

This is an interventional case series of 13 patients with orbital vascular malformations. These patients underwent sclerotherapy treatment with intralesional injection of pingyangmycin (Tianjin Taihe Pharmaceutical, Tianjin, China) at Fudan University Eye and ENT Hospital from September 2009 to April 2011. Patients with evident symptoms manifesting clinical findings that portend high risk for surgery, including lesions that are diffuse, deep in the orbital apex, with relative afferent papillary defect or without radiographic separation from the optic nerve, were included in this study. Informed consent was obtained from study subjects. Institutional review board approval was given for this study by the EENT hospital, which adhered to the tenets of the Declaration of Helsinki.

The clinical diagnosis was based upon clinical history, symptoms, clinical examinations and ultrasonography by ophthalmologists specialised in orbital diseases. In all cases, adjunctive CT and MRI were evaluated by radiologists with experience in analysing orbital lesions. When CT (Siemens AG, 
Berlin and München) or MRI (Siemens Verio V.3.0, German) was performed, patients were in the prone position to maximise the lesion size. Although CT uses ionising radiation, the anatomic relations of the vascular lesions to fixed, bony fiduciary landmarks provided by CT, was preferred by the orbital surgeons for performing surgery with sclerotherapy. Pertinent recorded clinical information was obtained, including age, sex, laterality, course of disease, presenting symptom, lesion location and diagnosis. Prior to sclerotherapy injections, all the patients underwent complete blood count; liver and renal function tests; chest radiographs; and electrocardiogram to exclude serious systemic diseases.

\section{Treatment procedure}

Patients with deep lesions had injection during incisional surgery to expose the tumours, whereas some with relative superficial but diffused lesions were treated with percutaneous injection as an outpatient. During surgery, patients sometimes underwent ipsilateral jugular vein compression to distend the tumour and maximise its size. The surgical approaches included anterior lateral approach, inferior sulcus approach and medial conjunctiva approach, depending on the location of the lesion.

Using a 21-gauge hypodermic needle, all lesions were punctured and blood or lymphatic fluid was withdrawn under direct visualisation. A $5 \mathrm{ml}$-syringe containing $2.0 \mathrm{ml}$ of pingyangmycin $(4 \mathrm{mg} / \mathrm{ml})$ in $0.9 \%$ (normal) saline was then used to repeatedly aspirate and irrigate the tumour lumens while altering the direction of the needle to displace blood while filling as much of the tumour as possible with the sclerosing drug solution. The total dosage of pingyangmycin was dependent on tumour size, usually ranging from $2-6 \mathrm{mg}(0.5-1.5 \mathrm{ml})$ with a maximum dose of $8 \mathrm{mg}(2 \mathrm{ml})$ for treatment. Direct visualisation of the injected agent in relation to the posterior margin of the treated tumour was maintained at all times to avoid posterior drainage into the cavernous sinus. To tamponade the needle track after sclerotherapy injection, a little bit of sodium chondroitin hyaluronate (Viscoat; Alcon, Ft. Worth, Texas, USA) was injected along the needle track and bipolar electric coagulation was applied to seal the track. Postoperatively, a compression bandage was placed for $24-48 \mathrm{~h}$ to prevent orbital haemorrhage. Twelve patients in this study had only one injection. None of the tumours were biopsied at the time of injection except for one patient who underwent a second injection and incisional biopsy to evaluate histopathologic changes caused by pingyangmycin.

\section{Evaluation of pingyangmycin effects on orbital vascular tumours}

To evaluate the efficacy of intralesional pingyangmycin, thinsection $(0.75 \mathrm{~mm})$ CT scans were performed before and after treatment to determine tumour size reduction. Tumour volumes were measured with CT image processing software (Syngo CT Workplace, Erlangen, Germany) applied to the 3-D reconstructed CT images. The volume of each tumour was calculated as the average of three separate measurements, each using three separate sets of tumour images. For the patient who underwent two injections, the postoperative proptosis and tumour volume we report were measured after the first injection.

To evaluate the histopathological alterations due to pingyangmycin treatment, we analysed paraffin sections of the open biopsy performed at the time of a second pingyangmycin injection in one patient. In addition to H\&E sections, collagen staining using Masson's trichrome stain and immunohistochemistry for factor VIII-related antigen, smooth muscle actin, vimentin, CD3, CD20, and CD68 were performed.

\section{Patient follow-up}

Patients were usually suggested to visit our outpatient clinic at 1 week, 2 months, 6 months and a year after the injection of pingyangmycin. Ocular and general physical examinations and preoperative adjunctive studies were performed to assess tumour response and possible local and systemic side effects due to pingyangmycin treatment.

\section{Statistical analysis}

Statistical analyses were performed using the Stata system (Stata V.10.0, StataCorp, College Station, Texas, USA). The matched $\mathrm{t}$ test was used to compare proptosis and tumour volume before and after therapy. The Wilcoxon rank sum (Mann-Whitney) test also was used to compare tumour volume reductions between the patients whose follow-up was greater than 6 months to those less than 6 months.

\section{RESULTS}

\section{Patient data}

Of the 13 patients, 5 were men and 8 were women. The patients' average age was 36 years (range, $16-58$ years). The average duration of disease prior to therapy was 31 months. Clinical diagnoses were venous malformation in six patients, cavernous haemangioma in six patients and lymphangioma in one patient. The most common presenting symptoms were proptosis, eyelid swelling, pain and double vision as listed in table 1. Distensibility with lowering of the head or Valsalva manoeuvre and phleboliths on CT images were peculiar to venous malformations. One patient underwent a percutaneous injection under local anaesthesia in the outpatient clinic, the others all underwent injections under direct observation after surgical exposure. The mean injected dose of pingyangmycin was $4.2 \mathrm{mg}$ (range 2-6 mg). The time interval between pingyangmycin injection and postoperative CT scans to assess treatment effects averaged 8.7 months (ranged 1.6-21.4 months), and the mean follow-up time 13.3 months (range, 2.3-23.4 months).

\section{Clinical effect and safety}

All patients reported significantly improved symptoms. The preoperative and postoperative proptosis averaged $3.4 \mathrm{~mm}$ (range, 0-6 mm) and $0.2 \mathrm{~mm}$ (range $-1.0-2.5 \mathrm{~mm}$ ), respectively. The proptosis reduction averaged $3.2 \mathrm{~mm}$ (range, $0-5.5 \mathrm{~mm}$ ) and was statistically significant $(p=0.001)$. Local swelling that occurred 3-5 days following sclerotherapy injections resolved spontaneously. One patient (Patient 1, table 1) developed deepening of the superior eyelid sulcus, whereas one developed mild enophthalmos (Patient 13, table 1). One patient with a large lesion (Patient 4, table 1), had some tumour reduction $(25 \%)$ after the first pingyangmycin injection (figure 1), and was given a second injection to enhance the therapeutic effect; this patient also had a $0.5 \mathrm{~cm}^{3}$ incisional biopsy at her second surgery. Her final tumour volume reduction was $64 \%$, and her proptosis reduction was $4.5 \mathrm{~mm}$, having measured $5.5 \mathrm{~mm}$ before treatment and $1.0 \mathrm{~mm}$ at her last visit. No patient experienced vision loss, nerve damage, scarring, necrosis, pulmonary fibrosis, cytopenia or anaphylaxis.

\section{Radiological analysis}

Eleven of the orbital vascular malformations were present in the muscle cone (85\%) and nine extended into the orbital apex (69\%). Preoperative tumour volume averaged $4.4 \pm 2.3 \mathrm{~cm}^{3}\left(1.6-8.8 \mathrm{~cm}^{3}\right)$, and postoperative volume averaged $1.5 \pm 1.7 \mathrm{~cm}^{3}\left(0.2-6.6 \mathrm{~cm}^{3}\right)$ after a single pingyangmycin injection (table 1$)$. The mean tumour 
Table 1 Clinical feature, treatment, and outcome of intralesional injection of pingyangmycin of the 13 patients with orbital vascular malformations

\begin{tabular}{|c|c|c|c|c|c|c|c|c|c|c|c|c|c|c|c|c|c|c|c|}
\hline \multirow[b]{2}{*}{ No. } & \multirow[b]{2}{*}{ Sex } & \multirow[b]{2}{*}{$\begin{array}{l}\text { Age } \\
\text { (year) }\end{array}$} & \multirow[b]{2}{*}{ Eye } & \multirow[b]{2}{*}{$\begin{array}{l}\text { Course of } \\
\text { disease } \\
\text { (months) }\end{array}$} & \multirow[b]{2}{*}{$\begin{array}{l}\text { Presenting } \\
\text { symptom }\end{array}$} & \multirow[b]{2}{*}{ Diagnosis } & \multicolumn{3}{|l|}{ Location } & \multirow[b]{2}{*}{$\begin{array}{l}\text { Dosage } \\
\text { (mg) }\end{array}$} & \multicolumn{3}{|c|}{ Proptosis } & \multicolumn{4}{|c|}{ Volume of lesion } & \multirow{2}{*}{$\begin{array}{l}\text { Interval } \\
\text { to post } \\
\text { imaging } \\
\text { (months) }\end{array}$} & \multirow{2}{*}{$\begin{array}{l}\text { Interval } \\
\text { to final } \\
\text { follow-up } \\
\text { (months) }\end{array}$} \\
\hline & & & & & & & $\begin{array}{l}\text { Muscle } \\
\text { cone }\end{array}$ & Quadrant & $\begin{array}{l}\text { Orbital } \\
\text { apex }\end{array}$ & & $\begin{array}{l}\text { Pre } \\
(\mathrm{mm})\end{array}$ & $\begin{array}{l}\text { Post } \\
\text { (mm) }\end{array}$ & $\begin{array}{l}\text { Reduction } \\
(\mathrm{mm})\end{array}$ & $\begin{array}{l}\text { Pre } \\
\left(\mathrm{cm}^{3}\right)\end{array}$ & $\begin{array}{l}\text { Post } \\
\left(\mathrm{cm}^{3}\right)\end{array}$ & $\begin{array}{l}\text { Reduction } \\
\left(\mathrm{cm}^{3}\right)\end{array}$ & $\begin{array}{l}\text { Reduction } \\
(\%)\end{array}$ & & \\
\hline 1 & $\mathrm{~F}$ & 38 & os & 0.3 & Slight pain & VM & Outer & $\mathrm{N}$ & No & 4.0 & 1.5 & 0.0 & 1.5 & 1.7 & 0.3 & 1.4 & 81.6 & 21.4 & 21.7 \\
\hline 2 & M & 52 & od & 2.0 & Proptosis, swelling & VM & Inner & $\mathrm{NI}$ & No & 4.0 & 6.0 & 0.5 & 5.5 & 3.8 & 2.5 & 1.3 & 34.6 & 1.6 & 21.2 \\
\hline 3 & $\mathrm{~F}$ & 39 & os & 36 & Proptosis, swelling & $\mathrm{CH}$ & Inner & $\mathrm{C}$ & Yes & 5.0 & 6.0 & 0.5 & 5.5 & 4.5 & 1.4 & 3.1 & 69.0 & 2.1 & 20.0 \\
\hline $4^{*}$ & $\mathrm{~F}$ & 16 & os & 84 & Proptosis, swelling & $\mathrm{CH}$ & Inner & s & Yes & 6.0 & 5.5 & 2.5 & 3.0 & 8.8 & 6.6 & 2.2 & 24.5 & 4.3 & 23.4 \\
\hline 5 & $\mathrm{~F}$ & 58 & od & 1.0 & Incidental CT finding & $\mathrm{CH}$ & Inner & C & Yes & 4.0 & 2.0 & 0.0 & 2.0 & 4.7 & 0.6 & 4.0 & 86.4 & 15.9 & 16.3 \\
\hline 6 & M & 28 & os & 0.8 & Proptosis, swelling & $\mathrm{CH}$ & Inner & C & Yes & 4.0 & 5.0 & 0.0 & 5.0 & 7.7 & 1.4 & 6.3 & 81.5 & 2.1 & 2.6 \\
\hline 7 & M & 36 & os & 0.7 & $\begin{array}{l}\text { Proptosis, Valsalva } \\
(+)\end{array}$ & VM & Inner & N & No & 6.0 & 3.0 & 0.0 & 3.0 & 4.8 & 1.8 & 3.0 & 61.7 & 8.8 & 9.1 \\
\hline 8 & M & 16 & od & 1.0 & Proptosis & VM & Inner & TS & Yes & 4.0 & 5.0 & 0.0 & 5.0 & 6.5 & 1.8 & 4.8 & 72.8 & 2.5 & 2.5 \\
\hline 9 & $\mathrm{~F}$ & 34 & od & 120 & $\begin{array}{l}\text { Proptosis, Valsalva } \\
(+)\end{array}$ & VM & Inner & TS & Yes & 2.0 & 3.0 & 0.0 & 3.0 & 1.7 & 0.5 & 1.2 & 70.6 & 1.9 & 2.5 \\
\hline 10 & $\mathrm{~F}$ & 48 & od & 0.5 & Slight pain & VM & Inner & NS & No & 4.0 & 0.0 & 0.0 & 0.0 & 1.6 & 0.2 & 1.4 & 88.3 & 17.2 & 17.5 \\
\hline 11 & $\mathrm{~F}$ & 42 & od & 10 & Slight fullness & $\mathrm{CH}$ & Inner & $C$ & Yes & 4.0 & 0.0 & 0.0 & 0.0 & 2.6 & 0.4 & 2.2 & 84.8 & 18.9 & 18.9 \\
\hline 12 & $\mathrm{~F}$ & 21 & os & 84 & Proptosis, strabismus & $\mathrm{L}$ & $\begin{array}{l}\text { Inner } \\
\text { and } \\
\text { outer }\end{array}$ & NS & Yes & 4.0 & 4.0 & 0.0 & 4.0 & 4.0 & 0.7 & 3.3 & 82.6 & 13.3 & 13.3 \\
\hline 13 & M & 41 & os & 60 & Proptosis, diplopia & $\mathrm{CH}$ & Inner & C & Yes & 4.0 & 3.5 & -1.0 & 4.5 & 5.1 & 1.4 & 3.6 & 71.9 & 3.0 & 4.5 \\
\hline
\end{tabular}

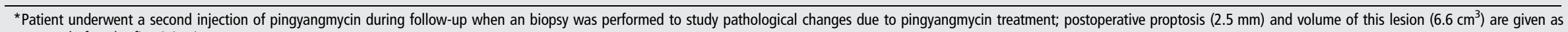

measured after the first injection.
C, central; $\mathrm{CH}$, cavernous haemangioma; I, inferior; L, lymphangioma; N, nasal; S, superior; T, temporal; VM, venous malformation. 

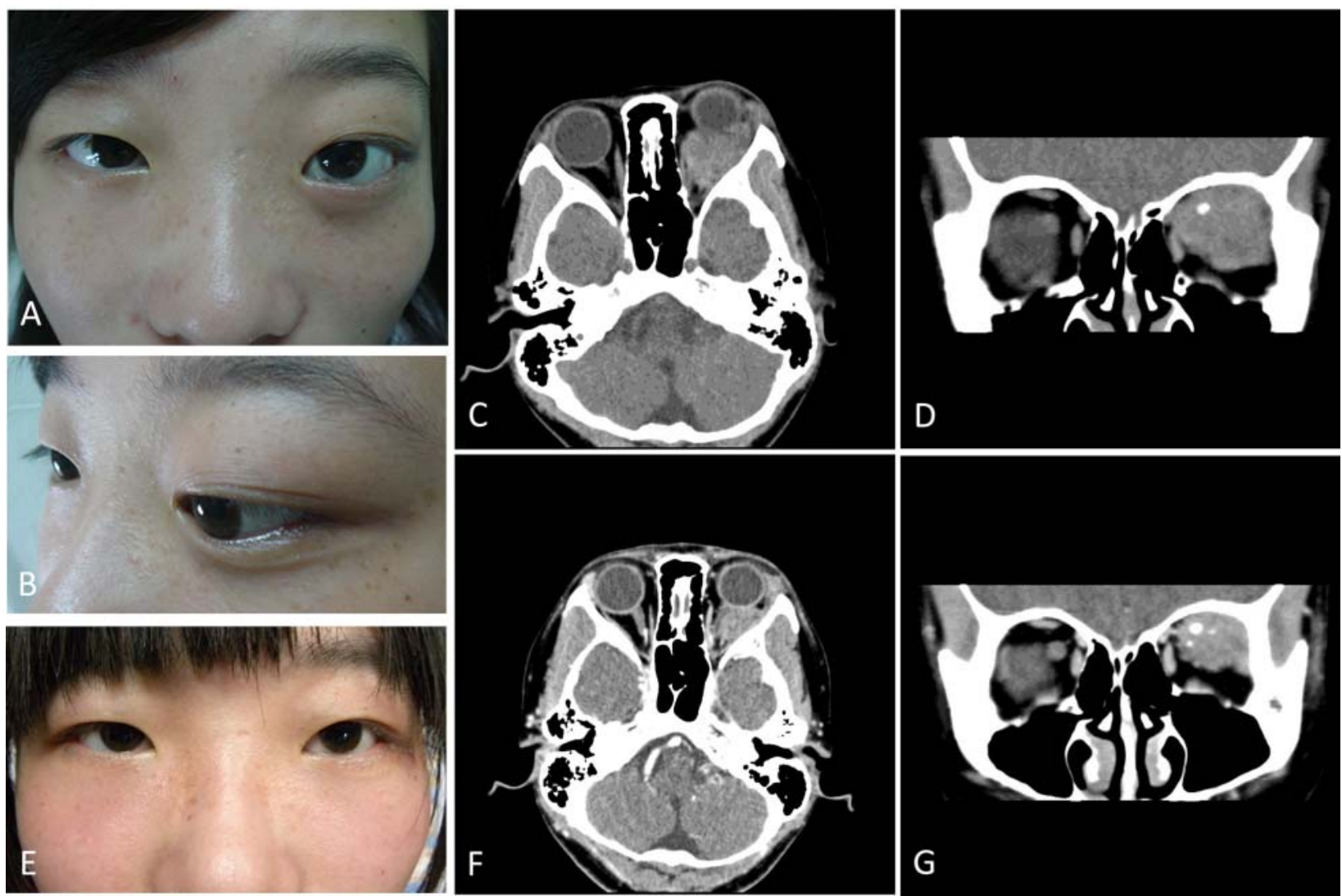

Figure 1 A 16-year-old girl with cavernous haemangioma demonstrating a $24.5 \%$ reduction of the tumour volume as measured by analysis of pretreatment and post-treatment $C T$ scans (case 4, table 1). $A, B$ and $E$ : the clinical appearance before $(A, B)$ and 4.3 months after (E) pingyangmycin injection; $C, D, F$ and $G$ : radiological images before $(C, D)$ and after $(F, G)$ injection.

volume reduction was $2.9 \pm 1.5 \mathrm{~cm}^{3}\left(1.2-6.3 \mathrm{~cm}^{3}\right)$ and the mean percentage of volume reduction was $70.0 \%(24.5-88.3 \%)$. The tumour volume reduction was statistically significant $(\mathrm{p}<0.001)$. The average tumour volume reduction for patients whose follow-up was greater than 6 months (nine patients, 74.2 $24.9 \%$ ) was not statistically different $(\mathrm{p}=0.758)$ from that of patients with less than 6 months follow-up (four patients, 68.2 $\pm 23.7 \%$ ).

\section{Histopathological analysis}

The tissue removed from Patient 4, approximately 4 months after pingyangmycin treatment, proved to be a portion of a cavernous haemangioma with treated and untreated areas (figure 2). The untreated areas showed typical large vascular spaces lined by attenuated endothelium and separated by fibrous septae (figure 2A). The treated portion of the tumour demonstrated thrombosis and occlusion of vascular lumens with small residual blood vessels. Scattered mononuclear leukocytes were present in areas of loose collagen, but not in areas showing denser fibrosis (figure 2B). Hemosiderin was present multifocally presumably due to old haemorrhage. The Masson trichrome stain showed increased amounts of collagen in the treated portion of the tumour (figure 2D). Immunohistochemical staining for Factor VIII-related antigen confirmed substantial loss of blood vessels (figure $2 \mathrm{~F}$ ), whereas vimentin and smooth muscle actin showed increased numbers of perivascular fibroblasts (figure $2 \mathrm{H}$ ) and myofibroblasts (figure 2J) following pingyangmycin treatment. There were numerous scattered CD3-positive T-lymphocytes (figure 3B), moderate numbers of CD68-positive histiocytes (figure $3 \mathrm{~F}$ ), and a few scattered CD20-positive B-lymphocytes (figure 3D), all in areas of loose collagen. All of these immunohistochemical findings were limited to the treated portion of the tumour and absent or sparse in the untreated portion of the tumour.

\section{DISCUSSION}

Vascular malformations represent a wide spectrum of pathological conditions with different clinical characteristics and natural evolutions. Initial differentiation of vascular malformations from other types of vascular tumours, based on differences in natural history, endothelial proliferation, and histology, was first proposed in 1982 by Mulliken and Glovacki. ${ }^{11}$ The International Orbital Society has adopted a new classification system for orbital vascular malformations based on the flow characteristics. ${ }^{9}$ In the system, vascular malformations are classified into: Type 1-no flow (lymphatic vascular malformations and some venous lymphatic lesions); Type 2-venous flow (distensible, nondistensible venous vascular malformations, and combined distensible venous-lymphatic vascular malformations); Type 3-arterial flow (arteriovenous malformation, cavernous haemangioma and acquired arteriovenous shunts). Obviously, arteriovenous malformation was not included in our study.

Orbital cavernous haemangiomas and venous and lymphatic malformations often lead to significant functional and cosmetic deficits. Although complete excision of a lesion is often the first choice for most oculoplastic surgeons, in the treatment of orbital vascular malformations, complete excision is often fraught with great risk for significant morbidity or is even impossible without damaging important structures. Thus, the alternative of sclerotherapy may be an attractive alternative for complex orbital malformations. 


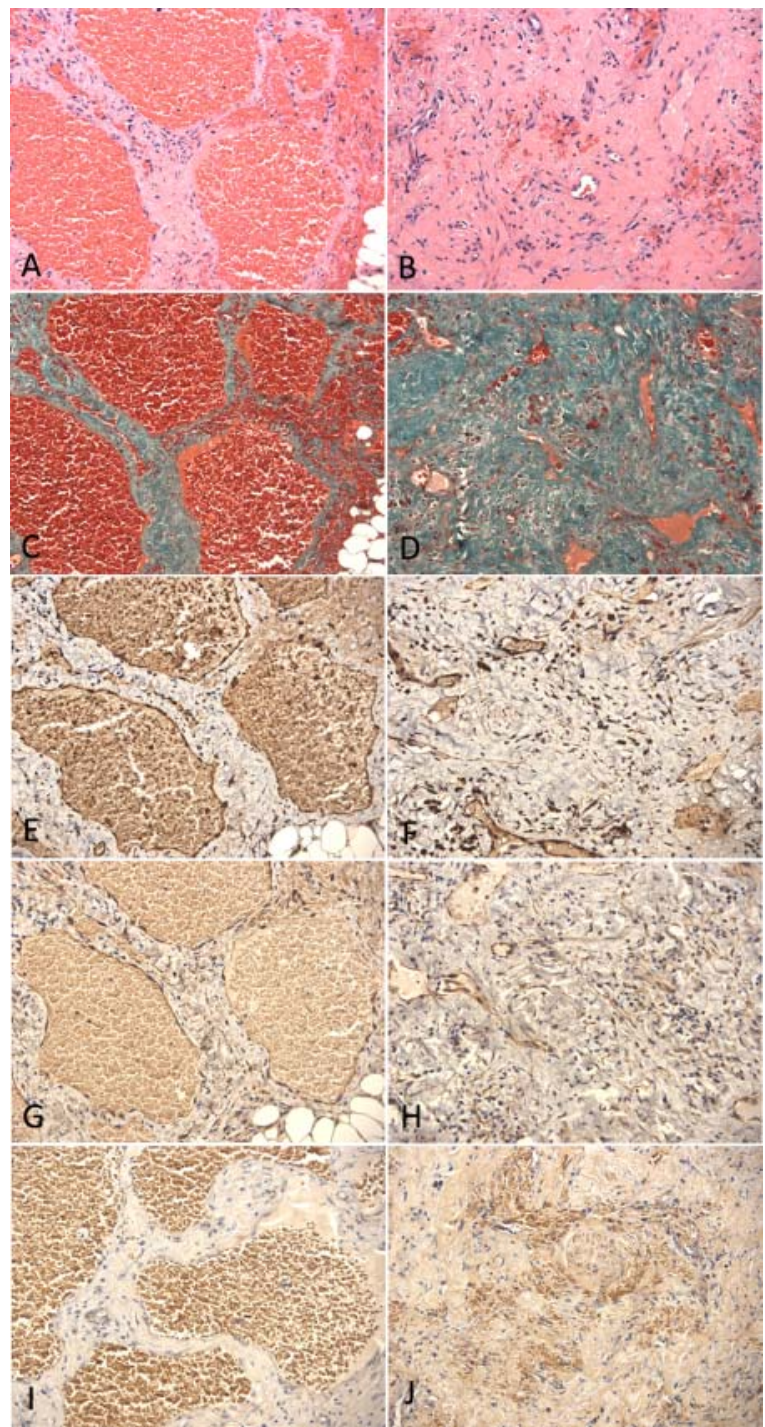

Figure 2 Fibrosis and immunohistopathologic changes of cavernous haemangioma after intralesional pingyangmycin in a 16-year-old girl with cavernous haemangioma. $A, C, E, G, I$ : untreated areas; $B, D, F, H, J$ : treated areas; $A, B$ : haematoxylin-eosin stain; $C, D$ : Masson trichrome stain; E,F: staining for Factor VIII-related antigen; G,H: staining for vimentin; I,J: staining for smooth muscle actin. Treated portion of tumour demonstrates collapsed vascular structures with luminal thrombosis and occlusion, perivascular fibrosis, and perivascular infiltration with mononuclear leukocytes (B), increased collagen deposition (D), reduced numbers of endothelial cells (F), and increased numbers of fibroblasts $(\mathrm{H})$ and myofibroblasts $(\mathrm{J})$. All original magnifications $\times 200$.

The first clinical trial of pingyangmycin as a sclerosing agent for venous malformations of the trunk was reported in $1991 .^{12}$ Since that time, it has been used successfully to treat a variety of lesions including those of the head and neck. ${ }^{5}$ In our study, all of our 13 patients were at high risk for complications from attempted excisional surgery for deep orbital vascular lesions and underwent successful intralesional injection of pingyangmycin without surgery or with only surgical exposure of the tumour. Many of these lesions presented a high risk of haemorrhage for percutaneous injection. Therefore, these patients underwent surgical exposure of the tumour to ensure precise localisation of the pingyangmycin injection and for control of haemorrhage.
In all cases, patients noted significant post-treatment reductions of proptosis, swelling, pain and other symptoms without complications. The reduction in lesional volume, when quantified by CT imaging, averaged $70 \%$ and was comparable to reductions of $75 \%$ reported for treatment of similar lesions at other sites in the head and neck. ${ }^{5} 13$ Our findings also compare favourably with those of Chen et al ${ }^{14}$ and Shen et al. ${ }^{15}$ Chen et $a l^{14}$ in 2008 reported results of the only other series of pingyangmycintreated orbital vascular malformations in the medical literature. They found resolution of proptosis, swelling and pain in 17 of 19 patients without complications when pingyangmycin lipiodol emulsion under fluoroscopic guidance was used as the sclerosing agent for venous malformations. In 2012, Shen et al ${ }^{15}$ reported satisfactory outcomes after bleomycin (another drug similar to bleomycin A5) injection for the treatment of orbital lymphatic venous malformation under percutaneous fluoroscopic guidance. Compared with these two studies, ours may be safer by avoiding the need for fluoroscopic guidance. In addition, our measurements of reduction in lesion volume are reliable, but the use of CT imaging, compared to MRI T- 2 weighted images, may underestimate measurements of vascular malformations, portions of which may be isodense to other soft tissue on the CT scan.

For complicated cavernous haemangiomas and venous and lymphatic malformations of the orbit, we prefer intralesional pingyangmycin to complete resection, especially when patients manifest clinical findings that portend high risk for surgery, including lesions that are diffuse or located at apex without radiographic separation from the optic nerve. Other high-risk signs include relative afferent pupillary defect upon compressing the lesion or obvious increases in size with Valsalva manoeuvre.

None of our 13 patients developed complications from their intralesional injections of pingyangmycin. Following injections, most of our patients experienced swelling that lasted for several days and then resolved spontaneously. Experience using pingyangmycin as a chemotherapeutic drug, has also shown it to be of relatively low toxicity. Due to the well-known toxicity of bleomycin, pulmonary fibrosis is a potential risk for patients receiving more than $160 \mathrm{mg}$ of bleomycin systemically. ${ }^{16}{ }^{17}$ In our study, the agent was injected intralesionally with a total dose of less than $8 \mathrm{mg}$, except for one patient who received two injections, separated by 4 months. No patient developed fever, pulmonary fibrosis, or other systemic complications and none exhibited local complications of tissue ulceration or optic nerve dysfunction.

Pingyangmycin, termed bleomycin A5, is refined from other components of bleomycin, and is isolated from Streptomyces pingyangensisn. It has a similar chemical structure to bleomycin, but the terminal amine is different. Intralesionally injected pingyangmycin brings the drug into direct contact with the endothelial lining and destroys the endothelial cells, resulting in sclerostenosis of the lumen. ${ }^{18}$ Like bleomycin, which promptly induces vascular endothelial cell apoptosis via the extrinsic pathway, ${ }^{19} 20$ it produces a 'devascular effect' which is a doseand time-dependent phenomenon characterised by partial intimal detachment followed by thrombosis within affected

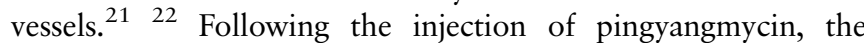
endothelial cells become swollen and vacuolised, the vascular intima is disrupted and the vascular walls become collapsed and thickened leading to narrowing or occlusion of the vessels. $^{20} 2223$ Inasmuch as our patients followed for less than 6 months post-treatment exhibited tumour reductions that were not statistically different from those followed for more than 6 months, it is likely that pingyangmycin causes rapid reduction in tumour volume on a timescale consistent with that reported for experimental models. 

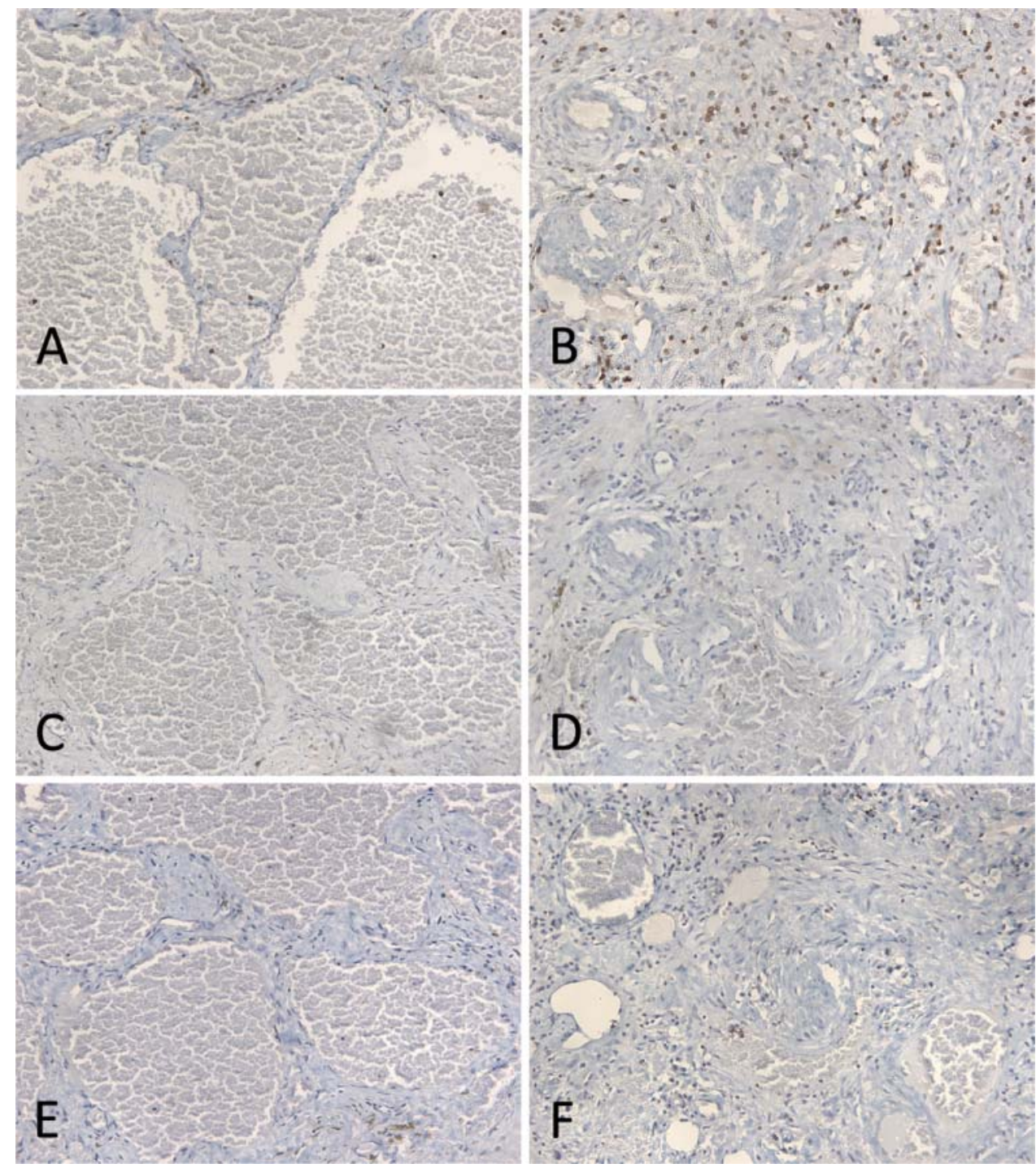

Figure 3 Immunohistochemical demonstration of mononuclear leukocytes in cavernous haemangioma after intralesional pingyangmycin. $A, C, E$ : untreated areas; B,D,F: treated areas; $A, B$ : stain for CD3; C,D: stain for CD20; E,F: stain for CD68. Treated areas show numerous scattered T-cells (B), few scattered B-cells $(D)$, and moderate numbers of mononuclear phagocytes $(F)$, which are largely absent in untreated portion of tumour. Original magnifications $\times 200$.

This is the first detailed histopathologic description of the effects of pingyangmycin or bleomycin on an orbital vascular malformation of any type. The partially excised cavernous haemangioma, 4 months after intralesional pingyangmycin, demonstrated collapsed thickened vascular walls with occlusion of lumina. This was associated with loss of endothelial cells as demonstrated by significantly reduced Factor VIII-related antigen staining in treated portions of the lesion. These findings are consistent with the known effects of pingyangmycin, which arrests the G2 and S phases of endothelial cells by causing breakage of DNA strands and inhibition of DNA repair by DNA ligase. $^{24} 25$ We also found perivascular chronic inflammation, principally of T-lymphocytes, but also containing mononuclear phagocytes and B-lymphocytes, findings similar to those that have been found in bleomycin-induced toxicity of the skin and lung. ${ }^{26-28}$ The predominance of T-lymphocytes reflects their importance in the evolving lesion. Recent studies of bleomycin toxicity have shown that the agent causes a T-lymphocyte-driven response, which begins as TH1-proinflammatory response and transforms to a TH2/TH17 anti-inflammatory and profibrotic response. ${ }^{29}$ Key drivers include direct and indirect stimulation of toll like receptor 2 on T-lymphocytes by bleomycin ${ }^{30}$ and immunosuppressive interleukin-6 and transforming growth factor- $\beta-1$-driven TH17 transformation of T-lymphocytes, ${ }^{31}$ which enhances soft tissue damage and subsequent fibrosis. ${ }^{32}$

The vascular alterations were also accompanied by perivascular fibrosis with increased numbers of fibroblasts and myofibroblasts, which have been described in bleomycin-induced lesions of the skin and lung. ${ }^{26} 27$ These connective tissue cellular and matrix alterations appear to be dependent upon cooperative signalling of monocyte chemoattractant protein-1 and transforming growth factor- $\beta 2$ in myofibroblast transformation and collagen expression. ${ }^{26} 2833$ It is likely that the combination of myofibroblast-associated matrix contraction and collagen deposition and crosslinking contributed to the substantial reductions in lesional volume that we measured by CT analysis.

In conclusion, intralesional pingyangmycin in the treatment of complicated orbital vascular malformations appears to be a safe and effective alternative therapy in challenging cases. 
Contributors Involved in design and conduct of study (JQ); collection and management of the data (HY, JG, YFY, RZ, QG); provision of patients and resources (HY, JQ, VME, JG, YFY); analysis and interpretation of the data (HY, JQ, VME); preparation of manuscript $(\mathrm{HY}, J \mathrm{Q}, J G)$; and review and approval of the manuscript (HY, JQ, VME, JG, YFY, RZ, QG)

Competing interests None.

Patient consent Obtained.

Ethics approval The study protocol was approved by the institutional review board of Eye and ENT Hospital of Fudan University, Shanghai, China.

Provenance and peer review Not commissioned; externally peer reviewed.

\section{REFERENCES}

1 Arat YO, Mawad ME, Boniuk M. Orbital venous malformations: current multidisciplinary treatment approach. Arch Ophthalmol 2004;122:1151-8.

2 Puttgen KB, Pearl M, Tekes A, et al. Update on pediatric extracranial vascular anomalies of the head and neck. Childs Nerv Syst 2010:26:1417-33.

3 Enjolras O, Wassef M, Chapot R. Introduction: ISSVA Classification. In: Enjolras O, Wassef M, Chapot R, eds. In: Color Atlas of Vascular Tumors and Vascular Malformations. New York: Cambridge University Press, 2007: 1-11.

4 Dubois J, Garel L. Imaging and therapeutic approach of hemangiomas and vascular malformations in the pediatric age group. Pediatr Radiol 1999;29:879-93.

5 Zheng JW, Yang XJ, Wang YA. Intralesional injection of pingyangmycin for vascular malformations in oral and maxillofacial regions: an evaluation of 297 consecutive patients. Oral Oncology 2009;45:872-6.

6 Chen WL, Yang ZH, Bai ZB. A pilot study on combination compartmentalization and sclerotherapy for the treatment of massive venous malformations of the face and neck. J Plast, Reconstr Aesthet Surg 2008;61:1486-92.

7 Pienaar C, Graham R, Geldenhuys S. Intralesional bleomycin for the treatment of hemangiomas. Plast Reconstr Surg 2006;117:221-6.

8 Mathur NN, Rana I, Bothra R. Bleomycin sclerotherapy in congenital lymphatic and vascular malformations of head and neck. Int J Ped Otorhinolaryngol 2005:69:75-80.

9 Rootman J. Diseases of the orbit: a multidisciplinary approach. 2nd edn. Philadelphia: Lippincott Williams \& Wilkins, 2003: 509-10.

10 Font RL, Croxatto JO, Rao NA. Tumors of the eye and ocular adnexa. AFIP Atlas of Tumor Pathology, 4th Series, Fascicle 5. WashingtonDC: American Registry of Pathology, 2006: 528-9.

11 Mulliken JB, Glowacki J. Hemangiomas and vascular malformations in infants and children:s classification based on endothelial characteristics. Plast Reconstr Surg 1982;69:412-20.

12 Zheng QT. Local injection of bleomycin A 5 in children with hemangiomas: analysis of 210 cases [in Chinese]. Chin J Surg 1991;29:290-1.

13 Zhang JW, Chen CJ, Zhang ZY. Intralesional injection of pingyangmycin for hemangiomas and vascular malformations in oral and maxillofacial region: a systematic review of the Chinese literature [in Chinese]. China J Oral Maxillofacial Surg 2003;1:102-5.

14 Chen Y, Li Y, Zhu Q, et al. Fluoroscopic intralesional injection with pingyangmycin lipiodol emulsion for the treatment of orbital venous malformations. Am J Radiol 2008;190:966-71.
15 Shen CY, Wu MC, Tyan YS, et al. Preliminary experience of percutaneous intralesional bleomycin injection for the treatment of orbital lymphatic-venous malformation refractory to surgery. Clin Radiol 2012:67:182-4.

16 Baskin D, Tander B, Bankaoglu M. Local bleomycin injection in the treatment of lymphangioma. Eur J Pediatr Surg 2005;15:383-6.

17 Wu HB, Lu GC. Complications of pingyangmcin:a review [in Chinese]. Chin J Clin Pharm 2001;10:53-5.

18 Gao Q, Wang C, Wen Y. An experimental study on effects of pingyangmycin on vessels [in Chinese]. Hua Xi Kou Qiang Yi Xue Za Zhi 2001;19:184-7.

19 Mungunsukh O, Griffin AJ, Lee YH, et al. Bleomycin induces the extrinsic apoptotic pathway in pulmonary endothelial cells. Am J Physiol Lung Cell Mol Physiol 2010:298:L696-703.

20 Wang C, Liu J, Pan W, et al. Pingyangmycin loaded bovine serum albumin microspheres for chemoembolization therapy: in vitro and in vivo studies. Int $J$ Pharm 2008;351:219-26.

21 Zeng QL, Li YH, Chen Y, et al. The experimental study of intra-arterial infusion of pingyangmycinlipiodol emulsion in rabbits [in Chinese]. J Clin Radiol 2000;19:376-9.

22 Kong WD, Li YH, Zeng QL, et al. Effect of pingyangmycin on the growth and cell cycle of human vascular endothelial cells [in Chinese]. Di Yi Jun Yi Da Xue Xue Bao 2003:23:830-2, 6.

23 Zhao JH, Zhao YF, Chen XM. Histological investigation of veins and venous malformations after injection of sclerosing agents. Asian J Oral Maxillofac Surg 2002;14:226-31.

24 Burger RM, Peisach J, Horwitz SB. Activated bleomycin. A transient complex of drug, iron, and oxygen that degrades DNA. J Biol Chem 1981; 256:11636-44.

25 Tai KW, Chang YC, Chou LS, et al. Cytotoxic effect of pingyangmycin on cultured KB cells. Oral Oncol 1998:34:219-23.

26 Yamamoto T. The bleomycin-induced scleroderma model: what have we learned for scleroderma pathogenesis? Arch Dermatol Res 2006;297:333-44.

27 Zhang K, Rekhter MD, Gordon D, et al. Myofibroblasts and their role in lung collagen gene expression during pulmonary fibrosis. A Combined immunohistochemical and in situ hybridization study. Am J Pathol 1994;145:114-25.

28 Yamamoto T, Nishioka K. Role of monocyte chemoattractant protein-1 and its receptor, CCR-2, in the pathogenesis of bleomycin-induced scleroderma. J Invest Dermatol 2003;121:510-16.

29 Liu HZ, Yang HZ, MI S, et al. Toll like receptor 2 mediates bleomycin-induced acute lung injury, inflammation and fibrosis in mice. Yao Xue Xue Bao (Acta Pharmaceutica Sinica) 2010;45:976-86.

30 Yang HZ, Cui B, Liu HZ, et al. Targeting TLR2 attenuates pulmonary inflammation and fibrosis by reversion of suppressive immune microenvironment. J Immunol 2009; 182:692-702.

31 Dong Z, Tai W, Yang Y, et al. The role of all-trans retinoic acid in bleomycin-induced pulmonary fibrosis in mice. Exp Lung Res 2012;38:82-9.

32 Wilson MS, Madala SK, Ramalingam TR, et al. Bleomycin and IL-1 $\beta$-mediated pulmonary fibrosis is IL-17A independent. J Exp Med 2010;207:535-52.

33 Gharaee-Kermani M, McCullumsmith RE, Charo IF, et al. CC-chemokine receptor 2 required for bleomycin-induced pulmonary fibrosis. Cytokine 2003; 24:266-76 